\title{
FRÉCHET DIFFERENTIABLE FUNCTIONALS AND SUPPORT POINTS FOR FAMILIES OF ANALYTIC FUNCTIONS \\ BY
}

\author{
PAUL C. COCHRANE AND THOMAS H. MACGREGOR
}

\begin{abstract}
Given a closed subset of the family $S^{*}(\alpha)$ of functions starlike of order $\alpha$ of a particular form, a continuous Fréchet differentiable functional, $J$, is constructed with this collection as the solution set to the extremal problem $\max \operatorname{Re} J(f)$ over $S^{*}(\alpha)$. Similar results are proved for families which can be put into one-to-one correspondence with $S^{*}(\alpha)$.

The support points of $S^{*}(\alpha)$ and $K(\alpha)$, the functions convex of order $\alpha$, are completely characterized and shown to coincide with the extreme points of their respective convex hulls. Given any finite collection of support points of $S^{*}(\alpha)$ (or $K(\alpha)$ ), a continuous linear functional, $J$, is constructed with this collection as the solution set to the extremal problem $\max \operatorname{Re} J(f)$ over $S^{*}(\alpha)$ (or $\left.K(\alpha)\right)$.
\end{abstract}

1. Introduction. Let $A$ denote the set of functions that are analytic in the open unit disk $\Delta=\{z:|z|<1\}$ in the complex plane C. The topology on $A$ which concerns us is that given by sequential convergence that is uniform on compact subsets of $\Delta$. By a continuous, linear functional on $A$ we mean a complex-valued functional defined on $A$ that is linear and continuous. In other words, if $J$ is such a functional then

$$
J(a f+b g)=a J(f)+b J(g)
$$

where $a, b \in \mathbf{C}$ and $f, g \in A$. Also, if $f_{n} \in A(n=1,2, \ldots)$ then

$$
f_{n} \rightarrow f \text { implies that } J\left(f_{n}\right) \rightarrow J(f) .
$$

We are also interested in continuous functionals which are Fréchet differentiable. In the context of families of analytic functions, this concept was introduced by J. A. Hummel in [13], where he uses the term "linear in the small". We shall reformulate Hummel's definition so that it depends on variations over the family. Suppose that $F \subset A$ and $f \in F$. Then, by a variation of $f$ we mean a function $f^{*}$, depending on a complex parameter $\varepsilon$, so that $f^{*} \in F$ and $f^{*}=f+g \cdot \varepsilon+o(\varepsilon)$. The function $g \in A$ and $O(\varepsilon)$ denotes a

Received by the editors July 21, 1975 and, in revised form, April 8, 1976.

AMS (MOS) subject classifications (1970). Primary 30A32, 30A38, 30A40, 30A76.

$K e y$ words and phrases. Analytic function, continuous linear functional, Fréchet differentiable functional, univalent function, starlike mapping, starlike function of order $\alpha$, convex mapping. convex function of order $\alpha$, variations of functions, bounded functions, support point, extreme point, convex hull, coefficient region, function with a positive real part, subordination. 
function of the variable $(z, \varepsilon)$ so that $o(\varepsilon) / \varepsilon \rightarrow 0$ as $\varepsilon \rightarrow 0$ when $z$ is in any compact subset of $\Delta$. The dependence of $f^{*}$ on $\varepsilon$ is given by a map from some subset of a neighborhood of $\varepsilon=0$ to $F$, and limits are meant in the usual restricted way.

Suppose that $J$ is a complex-valued functional defined on $F$. Then $J$ is called Fréchet differentiable at $f$ relative to $F$ if $f \in F$, and if to each variation of $f$ given by $f^{*}=f+g \cdot \varepsilon+o(\varepsilon)$, we conclude that

$$
J\left(f^{*}\right)=J(f)+\varepsilon I(f, g)+o(\varepsilon) .
$$

Moreover, we require that $I(f, \cdot)$ is a continuous, linear functional on $A$, and now $O(\varepsilon)$ denotes a function of $\varepsilon$ so that $o(\varepsilon) / \varepsilon \rightarrow 0$ as $\varepsilon \rightarrow 0$.

The study of Fréchet differentiation for a mapping between two Banach spaces has a long literature (see [8, pp. 92ff.] and [12, pp. 109ff.]). The definition prescribed through equation (3) is an adaption of this idea. It is different from the usual one primarily because the topology on $A$ cannot be given by a norm [26, p. 150].

Recently W. E. Kirwan and G. Schober [14] also considered Fréchet differentiable functionals in relation to families of analytic functions. In their paper, as in Hummel's, extremal problems are studied which are given by such functionals over the family of starlike mappings or related families.

Let $S^{*}(\alpha)$ denote the subset of $A$ consisting of starlike functions of order $\alpha$. Recall that this means that if $f \in S^{*}(\alpha)$ then $f(0)=0, f^{\prime}(0)=1$ and

$$
\operatorname{Re}\left\{z f^{\prime}(z) / f(z)\right\}>\alpha \quad(|z|<1) .
$$

This class was introduced by M. S. Robertson [22]. We note that $\alpha<1$ and $S^{*}=S^{*}(0)$ consists of the univalent mappings of $\Delta$ for which $f(\Delta)$ is starlike with respect to the origin.

The theorem stated below was proved by Kirwan and Schober [14]. It is slightly different from a result proved by Hummel [13] for the class $S^{*}$.

THEOREM A. Let $J$ be a continuous functional on $S^{*}(\alpha)$ and let

$$
\operatorname{Re} J\left(f_{0}\right)=\max \left\{\operatorname{Re} J(f): f \in S^{*}(\alpha)\right\}
$$

where $f_{0} \in S^{*}(\alpha)$. Suppose that $J$ has the Fréchet derivative $I$ at $f_{0}$ relative to $S^{*}(\alpha)$ and suppose that I does not have the form

$$
I\left(f_{0}, h\right)=a h(0)+b h^{\prime}(0) .
$$

Then $f_{0}$ has the form

$$
f_{0}(z)=z / \prod_{k=1}^{n}\left(1-x_{k} z\right)^{t_{k}}
$$

where $n$ is a positive integer and

$$
\left|x_{k}\right|=1, \quad t_{k}>0, \quad \sum t_{k}=2(1-\alpha) \quad(k=1,2, \ldots, n) .
$$


The condition that a functional be continuous and Fréchet differentiable (even on $A$ ) is quite general and so Theorem $\mathrm{A}$ applies to a large collection of extremal problems. For example, $J$ could be an analytic function of a finite number of continuous, linear functionals. This includes analytic functions of the coefficients in the power series of a function in $A$.

Theorem 4 in this paper is a converse of Theorem A. It asserts that any closed set of functions each of which has the form prescribed by equations (7) and (8) for a fixed positive integer $n$ is the set of solutions to (5) over $S^{*}(\alpha)$ for a suitable continuous, Fréchet differentiable functional $J$ on $S^{*}(\alpha)$.

In order to prove Theorem 4 we first prove a similar result for the family of bounded functions in $A$. In the process, we establish other facts about extremal problems over the class of bounded functions. General results about Fréchet differentiable functionals can be derived for several families of functions once proved for a representative family by means of suitable one-to-one correspondences between the families. This kind of transfer of information was utilized by Kirwan and Schober in [14].

A function $f$ is called a support point of a compact subset $F$ of $A$ if $f \in F$ and if there is a continuous, linear functional $J$, nonconstant on $F$, such that

$$
\operatorname{Re} J(f)=\{\max \operatorname{Re} J(g): g \in F\} .
$$

Each continuous, linear functional is Fréchet differentiable. Thus, an immediate consequence of Theorem $A$ is the fact that each support point of $S^{*}(\alpha)$ has the form given by equations (7) and (8). This was pointed out by Kirwan and Schober in [14]. We shall prove that the support points of $S^{*}(\alpha)$ require only one factor in this representation. That is, the support points of $S^{*}(\alpha)$ have the form

$$
f(z)=z /(1-x z)^{2(1-\alpha)} \quad(|x|=1) .
$$

The set of functions defined by equation (10) is also the set of extreme points of the closed convex hull of $S^{*}(\alpha)[4, \mathrm{p} .417]$. In general, the solution set to equation (9) contains an extreme point (when $J$ is a continuous, linear functional and $F$ is compact). This fact has been used as a means of solving extremal problems over various families (for example, see [2], [4], [15], [17]). For some classes, such as the functions with a positive real part or the typically real functions (see [3], [5], [21, p. 30] and [23]), the set of extreme points is quite smaller than the set of support points. For other classes, such as $S^{*}(\alpha)$ and the set of univalent functions with real coefficients [3, p. 102], the two sets are the same.

The determination of the support points for $S^{*}$ was obtained in [3], and the argument introduced there enables us to treat the class $S^{*}(\alpha)$. We also prove a converse result that shows that any finite set of functions of the form (10) is the solution set to equation (9) for a suitable continuous, linear functional $J$. 
Equivalent facts are derived for the set of convex functions of order $\alpha$, which we denote by $K(\alpha)$. Recall that $f \in K(\alpha)$ if $f \in A, f(0)=0, f^{\prime}(0)=1$ and

$$
\operatorname{Re}\left\{z f^{\prime \prime}(z) / f^{\prime}(z)+1\right\}>\alpha \quad(|z|<1) .
$$

It follows that $\alpha<1$ and $K=K(0)$ consists of the convex, univalent mappings of $\Delta$.

2. Bounded, analytic functions. Let $B$ denote the set of functions $\phi$ in $A$ so that

$$
|\varphi(z)|<1 \quad(|z|<1) .
$$

We shall present some facts about the coefficient regions for $B$ which are useful in discussing extremal problems. The initial results developed here are not new, and so we only sketch some of the arguments. These ideas were initiated by J. Schur [24], [25]. More recent accounts of facts about $B$ and references to the literature can be found in [1, pp. 101ff.]; [7, pp. 446ff.]; [11, pp. 497ff.]; [18].

Suppose that $\varphi \in B$ and

$$
\phi(z)=\sum_{n=0}^{\infty} c_{n} z^{n} \quad(|z|<1) .
$$

The $n$th coefficient region for $B$, denoted $B_{n}$, is the set of all $n$-tuples $\left(c_{0}, c_{1}, \ldots, c_{n-1}\right)$ such that there exists some function $\phi$ in $B$ so that the first $n$ coefficients of $\phi$ are the numbers $c_{0}, c_{1}, \ldots, c_{n-1}$. Properties of the class $B$ imply that $B_{n}$ is a compact, convex subset of $\mathbf{C}^{n}$.

Schur has given a characterization of functions in $B$ which correspond to points on $\partial B_{n}$ [24], [25]. The "Schur Algorithm" introduces the sequence of functions $\left\{\phi_{k}\right\}$ defined inductively by $\phi_{0}=\phi$ and

$$
\phi_{k}(z)=\frac{1}{z} \frac{\phi_{k-1}(z)-\phi_{k-1}(0)}{1-\overline{\phi_{k-1}(0)} \phi_{k-1}(z)} .
$$

If $\phi \in B$ then $\phi_{k} \in B$ for $k=0,1, \ldots$ and thus

$$
\left|\phi_{k}(0)\right|<1 \quad(k=0,1, \ldots) .
$$

Also, equality holds here for a given $k$ only when $\phi_{k}$ is a constant function (and then $\phi_{j}=0$ for $j>k$ ). The set of inequalities (15) is a necessary and sufficient condition on the coefficients $\left\{c_{n}\right\}$ so that $\phi \in B[7$, p. 446]. It follows that the inequality $\left|\phi_{n-1}(0)\right|<1$ defines the set $B_{n}$ and the equality $\left|\phi_{n-1}(0)\right|=1$ defines $\partial B_{n}$.

Suppose that $\phi \in B,\left|\phi_{k}(0)\right|<1$ for $k=0,1, \ldots, j-1$ and $\left|\phi_{j}(0)\right|=1$. Then $\phi_{j}$ is a constant and, by reversing equation (14) for $k=j, j-$ $1, \ldots, 1,0, \phi$ is determined. The dependence of $\phi_{k-1}(0)$ only on $c_{0}, c_{1}, \ldots, c_{k-1}, \bar{c}_{0}, \bar{c}_{1}, \ldots, \bar{c}_{k-1}$, therefore, implies that if $c_{0}, c_{1}, \ldots, c_{j-1}$ are 
given and if $\phi_{j}(0)$ is given and subject to the condition $\left|\phi_{j}(0)\right|=1$, then $\phi$ is uniquely determined. As pointed out by Schur, such a function takes on the form

$$
\phi(z)=\left(\alpha_{0}+\alpha_{1} z+\cdots+\alpha_{j} z^{j}\right) /\left(\bar{\alpha}_{j}+\bar{\alpha}_{j-1} z+\cdots+\bar{\alpha}_{0} z^{j}\right) .
$$

We shall show that these functions also may be expressed in the form

$$
\phi(z)=x \prod_{k=1}^{j} \frac{z+\beta_{k}}{1+\bar{\beta}_{k} z}
$$

where

$$
|x|=1, \quad\left|\beta_{k}\right|<1 \quad(k=1,2, \ldots, j) .
$$

Functions given by equations (17) and (18) are characterized as those functions in $A$ that are continuous on $\bar{\Delta}$ and satisfy $|\phi(z)|=1$ for $|z|=1[6, p$. 12]. These properties hold for $\phi$ once $\phi_{j}$ is a constant of modulus 1 since these properties pass on through the sequence $\phi_{j}, \phi_{j-1}, \ldots, \phi_{1}, \phi_{0}$. This also shows that the number of factors in equation (17) must be $j$ as $\phi$ maps $\Delta j$ to 1 onto $\Delta[6$, p. 13]. A function defined by equation (17) and (18) shall be called a finite Blaschke product of degree $j$.

The set of functions $\phi$ associated with a point on $\partial B_{n}$ may be characterized by a more general form than equation (17) by allowing $n-1$ factors and replacing the condition $\left|\beta_{k}\right|<1$ by $\left|\beta_{k}\right|<1$. Any factor of the form $(z+$ $\beta) /(1+\bar{\beta} z)$, where $|\beta|=1$, is a constant of modulus one and may be absorbed in the $x$ term. We call such a function a finite Blaschke product of degree at most $n-1$.

We summarize these known facts by the following statement.

Lemma 1. The set $B_{n}$ is a compact, convex subset of $C^{n}$. The set $\partial B_{n}$ is in one-to-one correspondence with the set of finite Balschke products of degree at most $n-1$.

Each continuous, linear functional $J$ on $A$ is given by a sequence $\left\{b_{n}\right\}$ which satisfies

$$
\lim \sup \left|b_{n}\right|^{1 / n}<1
$$

and so that

$$
J(f)=\sum_{n=0}^{\infty} b_{n} a_{n}
$$

when

$$
f(z)=\sum_{n=0}^{\infty} a_{n} z^{n} \quad(|z|<1) \quad[27] .
$$

We shall show that when the sum in equation (20) is only a finite sum (and 
nontrivial) then there is a unique function in $B$ maximizing the functional $\operatorname{Re} J(f)$. This result is a simple consequence of Lemma 1 and the next lemma. The actual solution to extremal problems over $B$ has a long literature (see [9, p. 145], [11, pp. 497ff.], [18]).

LEMMA 2. The set of extreme points of $B_{n}$ is $\partial B_{n}$.

Proof. No interior point of a set can be an extreme point of that set. Thus, we need only show that each point of $\partial B_{n}$ is an extreme point of $B_{n}$. Suppose that $a \in \partial B_{n}$ and $a=t b+(1-t) c$ where $0<t<1, b \in B_{n}$ and $c \in B_{n}$. If either $b$ or $c$ is an interior point of $B_{n}$ then so is $a$. Thus, $b \in \partial B_{n}$ and $c \in \partial B_{n}$. Lemma 1 implies that the points $a, b$ and $c$ correspond uniquely to functions $\phi, \psi$ and $\omega$, respectively, each of which is a finite Blaschke product of degree at most $n-1$. The relation $a=t b+(1-t) c$ is therefore equivalent to $\phi=t \psi+(1-t) \omega$. The functions $\phi, \psi$ and $\omega$ are analytic in $\bar{\Delta}$ and have modulus equal to 1 on $\partial \Delta$. The relation $\phi(z)=t \psi(z)+(1-t) \omega(z)$ also holds for $|z|=1$. If $\alpha, \beta$ and $\gamma$ are complex numbers with $|\alpha|=1,|\beta|<1,|\gamma| \leqslant 1$ and if $\alpha=t \beta+(1-t) \gamma, 0<t<1$, then $\alpha=\beta=\gamma$. Therefore, $\phi(z)=$ $\psi(z)=\omega(z)$ for $|z|=1$ and so $\psi=\omega$. This implies that $b=c$, and, consequently, $a$ is an extreme point of $B_{n}$.

THEOREM 1. Let $n$ be a positive integer and let $b_{k}(k=0,1, \ldots, n-1)$ be complex numbers, at least one of which is not zero. Let the functional $J$ be defined by

$$
J(\phi)=\sum_{k=0}^{n-1} b_{k} c_{k}
$$

where $\phi(z)=\sum_{k=0}^{\infty} c_{k} z^{k}(|z|<1)$. Then there is a unique function $\phi_{0}$ in $B$ so that

$$
\operatorname{Re} J\left(\phi_{0}\right)=\max \{\operatorname{Re} J(\phi): \phi \in B\} .
$$

Moreover, $\phi_{0}$ is a finite Blaschke product of degree at most $n-1$.

Conversely, if $\phi_{0}$ is a finite Blaschke product of degree not exceeding $n-1$, then there is a functional $J$ of the form (21) so that $\phi_{0}$ is the unique function in $B$ satisfying equation (22).

Proof. We identify the $n$-dimensional complex plane $\mathbf{C}^{n}$ with the $2 n$-dimensional real Euclidean space $\mathbf{R}^{2 n}$ in the usual way. Namely, the point $\left(z_{1}, z_{2}, \ldots, z_{n}\right)$ in $\mathbf{C}^{n}$ corresponds to $\left(x_{1}, y_{1}, x_{2}, y_{2}, \ldots, x_{n}, y_{n}\right)$ where $x_{k}=$ $\operatorname{Re} z_{k}$ and $y_{k}=\operatorname{Im} z_{k}(k=1,2, \ldots, n) . B_{n}$ shall be regarded as a subset of $\mathbf{R}^{2 n}$ and the results about $B_{n}$ shall be used in this setting.

Suppose that $b_{k}$ and $J$ are prescribed by the initial hypotheses of this theorem. Let 


$$
\begin{gathered}
\alpha_{k}=\operatorname{Re} b_{k}, \quad \beta_{k}=\operatorname{Im} b_{k}, \quad x_{k}=\operatorname{Re} c_{k} \quad \text { and } \\
y_{k}=\operatorname{Im} c_{k} \quad(k=0,1, \ldots, n-1)
\end{gathered}
$$

and let $M=\max \{\operatorname{Re} J(\phi): \phi \in B\}$. Then, the condition $\operatorname{Re} J(\phi)<M$ is equivalent to

$$
\sum_{k=0}^{n-1}\left(\alpha_{k} x_{k}-\beta_{k} y_{k}\right)<M .
$$

If $\phi$ varies over $B$, then, as not all of the numbers $b_{k}$ are zero, inequality (23) defines a closed half-space in $\mathbf{R}^{2 n}$ which contains $B_{n}$. The set of points for which equality holds in (23) defines a support plane to $B_{n}$ which we denote by $P$. The set $P \cap B_{n}$ is a convex subset of $\partial B_{n}$. If $P \cap B_{n}$ contained two distinct points, say $b$ and $c$, then every point on the segment $t b+(1-t) c, 0$ $<t<1$, belongs to $\partial B_{n}$ and fails to be an extreme point of $B_{n}$. This is impossible because of Lemma 2 and so $P \cap B_{n}$ consists of a single point, say a. Since $a \in \partial B_{n}$ it corresponds uniquely to a function $\phi_{0}$ in $B$ which is a finite Blaschke product of degree not exceeding $n-1$. This function is the unique extremal function for equation (22).

Conversely, suppose that $\phi_{0}$ is a finite Blaschke product of degree at most $n-1$. Then $\phi_{0}$ corresponds to a point $a$ on $\partial B_{n}$. There is a supporting plane $P$ to $B_{n}$ through $a$. This plane may be expressed in the form

$$
\sum_{k=0}^{n-1}\left(\gamma_{k} x_{k}+\delta_{k} y_{k}\right)=M
$$

so that $B_{n}$ is contained in the closed half-space determined from equation (24) by replacing $=$ by $<$. If we let $b_{k}=\gamma_{k}-i \delta_{k}$ and define $J$ by equation (21), then $\phi_{0}$ is the unique function in $B$ for which (22) holds.

LEMMA 3. Let $C$ be a nonvoid, closed subset of $\mathbf{R}^{n}$. There is a real-valued infinitely differentiable function $\Phi$ defined on $\mathbf{R}^{n}$ so that $\Phi(x)=0$ if $x \in C$ and $\Phi(x)<0$ if $x \notin C$.

This lemma is proved in [10, p. 17]. We shall only need to take advantage of the differentiability of the function $\Phi$.

THEOREM 2. Let $n$ be a positive integer and let $F$ be a nonvoid, closed set of finite Blaschke products of degree at most $n-1$. Then there is a complex-valued functional $J$ which is continuous and Fréchet differentiable on $A$ and so that the equation

$$
\operatorname{Re} J\left(\phi_{0}\right)=\max \{\operatorname{Re} J(\phi): \phi \in B\}
$$

holds for a function $\phi_{0}$ in $B$ if and only if $\phi_{0} \in F$.

Proof. Let $\mu$ be the mapping of $A$ onto $\mathbf{C}^{n}$ defined by $\mu(\phi)=$ $\left(c_{0}, c_{1}, \ldots, c_{n-1}\right)$ where $\phi(z)=\sum_{k=0}^{\infty} c_{k} z^{k}(|z|<1)$. Because of Lemma 1 and 
the fact that $F$ is closed, the set $D=\mu(F)$ is a compact subset of $\partial B_{n}$ and $\mu(\phi) \notin D$ if $\phi \in B-F$. Let $\pi$ denote the usual map of $C^{n}$ onto $\mathbf{R}^{2 n}$ described in the proof of Theorem 1 and let $\mu^{*}=\pi \circ \mu$. Let $\Phi$ be a function given by Lemma 3 where $C=\pi(D)$ and let $J_{1}=\Phi \circ \mu^{*}$. Then $J_{1}$ is a real-valued continuous functional on $A, J_{1}(\phi)=0$ if $\phi \in F$ and $J_{1}(\phi)<0$ if $\phi \in B-F$.

Suppose that $f$ and $g$ belong to $A, f(z)=\sum_{k=0}^{\infty} a_{k} z^{k}$ and $g(z)=\sum_{k=0}^{\infty} b_{k} z^{k}$. Suppose that $f^{*}$ is a variation of $f$ of the form $f^{*}=f+\varepsilon g+o(\varepsilon)$. A simple computation depending on the differentiability of $\Phi$ shows that

$$
\begin{aligned}
J_{1}\left(f^{*}\right)= & J_{1}(f)+\gamma\left[\lambda_{1} \alpha_{0}+\lambda_{2} \beta_{0}+\cdots+\lambda_{2 n-1} \alpha_{n-1}+\lambda_{2 n} \beta_{n-1}\right] \\
& +\delta\left[-\lambda_{1} \alpha_{0}+\lambda_{2} \beta_{0}+\cdots-\lambda_{2 n-1} \alpha_{n-1}+\lambda_{2 n} \beta_{n-1}\right]+o(\varepsilon),
\end{aligned}
$$

where $\gamma=\operatorname{Re} \varepsilon, \delta=\operatorname{Im} \varepsilon, \alpha_{k}=\operatorname{Re} b_{k}, \beta_{k}=\operatorname{Im} b_{k}$ and $\lambda_{k}$ is the $k$ th partial derivative of $\Phi$ at the point in question.

Let the functional $J$ be defined on $A$ by the equation $J(f)=J_{1}(f)-$ $i J_{1}(i f)$. Then $J$ is continuous on $A$ and equation (26) implies that $J\left(f^{*}\right)=$ $J(f)+I(f, g) \varepsilon+o(\varepsilon)$ and $I(f, \cdot)$ is a continuous linear functional on $A$. Thus $J$ is Fréchet differentiable on $A$. Since $J_{1}=\operatorname{Re} J$, the conclusions of the theorem concerning equation (25) follow.

3. Solution sets for $S^{*}(\alpha)$ and other families. There are several families of analytic functions which may be put into a one-to-one correspondence with $B$ by means of simple analytic operations. This makes it possible to deduce results similar to Theorem 2 for any such family. Results similar to Theorem A also hold for these families since they also are in a suitable one-to-one correspondence with $S^{*}$. Each family has a distinguished form for the solution set. These correspond to the forms already singled out for $S^{*}(\alpha)$ and $B$.

Let $P$ denote the subset of $A$ of functions $p$ for which $\operatorname{Re} p(z)>0(|z|<1)$ and $p(0)=1$. A one-to-one correspondence between $B$ and $P$ is given by the formula

$$
p(z)=(1+, z \phi(z)) /(1-z \phi(z)),
$$

where $\phi \in B$ and $p \in P$.

LEMMA 4. Suppose that $p \in P, p$ is continuous on $\bar{\Delta}$ except at the points $y_{k}$ $(k=1,2, \ldots, n)$, where $p$ has a pole. Also, suppose that $\operatorname{Re} p(z)=0$ if $|z|=1$ and $z \neq y_{k}(k=1,2, \ldots, n)$. Then $p$ has the form

$$
p(z)=\sum_{k=1}^{n} t_{k} \frac{1+x_{k} z}{1-x_{k} z}
$$

where

$$
t_{k}>0, \quad\left|x_{k}\right|=1, \quad \sum t_{k}=1 \quad(k=1,2, \ldots, n)
$$


Proof. The pole of $p$ at $y_{k}$ must be a simple one, since any higher order pole would imply that there are values of $z$ in $\Delta$ (and sufficiently near $y_{k}$ ) for which $\operatorname{Re} p(z)<0$. Let $x_{k}=\bar{y}_{k}$. Then we may write

$$
p(z)=a_{k} /\left(1-x_{k} z\right)+p_{k}(z)
$$

where the function $p_{k}$ is analytic at $y_{k}$ and $a_{k} \neq 0$. The function $w=(1-$ z) $)^{-1}$ maps $\Delta$ one-to-one onto the region $\left\{w: \operatorname{Re} w>\frac{1}{2}\right\}$. This implies that $a_{k}$ is real and positive, because any other value of $a_{k}$ would contradict the condition $\operatorname{Re} p(z)>0(|z|<1)$ when $z$ is sufficiently close to $y_{k}$.

Let $t_{k}=a_{k} / 2$ and define the function $q$ by

$$
q(z)=\sum_{k=1}^{n} t_{k} \frac{1+x_{k} z}{1-x_{k} z}
$$

The function $s$ defined by $s(z)=p(z)-q(z)$ is analytic in $\Delta$ and because

$$
t_{k} \frac{1+x_{k} z}{1-x_{k} z}=\frac{a_{k}}{1-x_{k} z}-t_{k}
$$

$s$ is also continuous in $\bar{\Delta}$. If $|z|=1$ and $z \neq y_{k}(k=1,2, \ldots, n)$ then $\operatorname{Re} p(z)=0$ and $\operatorname{Re} q(z)=0$. Therefore, $\operatorname{Re} s(z)=0$ for all $z$ with $|z|=1$. This implies that $s$ is a constant and that the constant is purely imaginary. Since $s(0)=1-\sum_{k=1}^{n} t_{k}$ is real, $s$ must be the constant 0 . Therefore, $p=q$ and $\sum_{k=1}^{n} t_{k}=1$. This completes the proof.

A finite Blaschke product of degree $n(n>0)$ is mapped by the correspondence (27) onto a function in $P$ having the form described by equations (28) and (29). This is a consequence of the characterization of finite Blaschke products as the functions in $B$ that are continuous in $\Delta$ and satisfy $|\phi(z)|=1$ for $|z|=1$. These properties of $\phi$ correspond to the properties of the function $p$ in Lemma 4, if $\phi$ and $p$ are related by (27).

As is well known, the family $S^{*}$ is put into a one-to-one correspondence with $P$ through the relation

$$
p(z)=z f^{\prime}(z) / f(z) .
$$

In this correspondence, a function in $P$ described by equations (28) and (29) maps to the function in $S^{*}$ given by the equations

$$
f(z)=\frac{z}{\prod_{k=1}^{n}\left(1-x_{k} z\right)^{\lambda_{k}}}
$$

and

$$
\left|x_{k}\right|=1, \quad \lambda_{k}>0, \quad \Sigma \lambda_{k}=2 \quad(k=1,2, \ldots, n) .
$$

This depends on a computation based on relation (32) and where $\lambda_{k}=2 t_{k}$. The equation 


$$
g(z)=z[f(z) / z]^{1-\alpha}
$$

defines a one-to-one correspondence from the class $S^{*}$ to the class $S^{*}(\alpha)$. In this correspondence, a function of the form described by equations (33) and (34) maps to a function given by equations (7) and (8) of Theorem $A$.

THEOREM 3. Let $J$ be a continuous functional on $B$ and suppose that $\phi_{0} \in B$ and

$$
\operatorname{Re} J\left(\phi_{0}\right)=\max \{\operatorname{Re} J(\phi): \phi \in B\} .
$$

Also, let $J$ have a Fréchet derivative at $\phi_{0}$ relative to $B$ which is not the zero functional on $A$. Then $\phi_{0}$ is a finite Blaschke product.

Proof. Let $\mu$ and $\nu$ denote the mappings $\mu: B \rightarrow P$ and $\nu: P \rightarrow S^{*}$ defined by equations (27) and (32), respectively. The function $\sigma=\nu \circ \mu$ is a one-toone mapping of $B$ onto $S^{*}$. Also $\sigma$ is continuous on $B$ since $\mu$ and $\nu$ are continuous on $B$ and $P$, respectively. Let $J$ denote the functional described in this theorem and define the functional $I$ on $S^{*}$ by $I(f)=J\left(\sigma^{-1}(f)\right)$. Then $I$ is continuous on $S^{*}$.

Suppose that $\phi_{0} \in B$ and equation (36) holds. Let $f_{0}=\sigma\left(\phi_{0}\right)$. We shall show that $f_{0}$ satisfies all of the hypotheses of Theorem $\mathrm{A}$, with $\alpha=0$, with respect to the functional $I$. Equation (5) holds since it is equivalent to equation (36). We need to show that $I$ has a Fréchet derivative at $f_{0}$ relative to $S^{*}$.

Suppose that $f \in S^{*}$ and let $f^{*}$ be a variation of $f$ given by $f^{*}=f+\varepsilon g+$ $o(\varepsilon)$. This implies that $f^{\prime \prime}=f^{\prime}+\varepsilon g^{\prime}+o(\varepsilon)$ with the usual meaning for $o(\varepsilon)$. Let $\phi=\sigma^{-1}(f)$ and $\phi^{*}=\sigma^{-1}\left(f^{*}\right)$. Equations (32) and (27) allow $\phi^{*}(z)$ to be expressed in the form

$$
\phi^{*}(z)=\phi(z)+\frac{2\left[f(z) g^{\prime}(z)-g(z) f^{\prime}(z)\right]}{\left[z f^{\prime}(z)+f(z)\right]^{2}} \varepsilon+o(\varepsilon) .
$$

This equation may be more simply expressed as

$$
\phi^{*}=\phi+\Lambda(f, g) \in+o(\varepsilon)
$$

where $\Lambda(f, g)$ is initially defined on the set $\{g\}$ occurring through all variations of $f$. The normalizations of $S^{*}$ show that $\Lambda(f, g)$ is well defined for each $g$ in $A$ if $g(0)=0$. On this subset of $A, \Lambda(f, \cdot)$ is a continuous, linear operator.

Equation (38) defines a variation of $\phi$ with respect to the family $B$. If $J$ is Fréchet differentiable at $\phi$ with respect to $B$, then we may write

$$
\begin{aligned}
I\left(f^{*}\right) & =J\left(\sigma^{-1}\left(f^{*}\right)\right)=J\left(\phi^{*}\right)=J(\phi)+K[\phi, \Lambda(f, g)] \varepsilon+o(\varepsilon) \\
& =I(f)+L(f, g) \varepsilon+o(\varepsilon) .
\end{aligned}
$$


Here, $K(\phi, \cdot)$ is a continuous, linear functional on $A$ and therefore $L(f, \cdot)$ is a continuous, linear functional on $F$. We may extend this functional to a functional $\tilde{L}$ on $A$ by $\tilde{L}(f, g)=L(f, h)$, where $g \in A$ and $h(z)=g(z)-$ $g(0)$. Then $\tilde{L}(f, \cdot)$ is a continuous, linear functional on $A$, and so the relation $I\left(f^{*}\right)=I(f)+\tilde{L}(f, g) \varepsilon+o(\varepsilon)$ shows that $I$ is Fréchet differentiable at $f$ with respect to $S^{*}$.

In order to apply Theorem A, and thereby establish this theorem, it only remains to show that the functional $\tilde{L}\left(f_{0}, \cdot\right)$ does not have the form

$$
\tilde{L}\left(f_{0}, g\right)=a g(0)+b g^{\prime}(0) \text {. }
$$

Suppose that equation (39) holds. Then, in particular, $L\left(f_{0}, g\right)=a g(0)+$ $b g^{\prime}(0)$ for all $g$ in $F$. This is the same as $K\left[\phi_{0}, \Lambda\left(f_{0,}, g\right)\right]=b g^{\prime}(0)$ for all $g$ in $F$. The continuous linear functional $K\left[\phi_{0}, \cdot\right]$ is given by a sequence $\left\{b_{n}\right\}$ described by equations (19) and (20). If we write

$$
k(z)=\frac{2\left[f_{0}(z) g^{\prime}(z)-g(z) f_{0}^{\prime}(z)\right]}{\left[z f_{0}^{\prime}(z)+f_{0}(z)\right]^{2}}=\sum_{n=0}^{\infty} c_{n} z^{n},
$$

then we conclude that $\sum_{n=0}^{\infty} b_{n} c_{n}=b g^{\prime}(0)$ for every $g$ in $F$. By solving the differential equation defined with $k(z)=z^{n}(n=0,1,2, \ldots)$ we determine a suitable function $g$ belonging to $F$. Quite specifically, we may set

$$
g(z)=f_{0}(z)\left\{\int_{0}^{z} \frac{w^{n}\left[w f_{0}^{\prime}(w)+f_{0}(w)\right]^{2}}{2 f_{0}^{2}(w)} d w\right\} .
$$

For such a choice of $g$ we have $g^{\prime}(0)=0$ and so the condition $\sum_{n=0}^{\infty} b_{n} c_{n}=$ $b g^{\prime}(0)$ reduces to $b_{n}=0$. This implies that $K\left[\phi_{0}, \cdot\right]$ is the zero functional. This provides the contradiction.

THEOREM 4. Let $G$ be a closed subset of $A$ of functions each of which has the form

$$
f(z)=\frac{z}{\prod_{k=1}^{n}\left(1-x_{k} z\right)^{i_{k}}}
$$

where $n$ is a given positive integer and

$$
\left|x_{k}\right|=1, \quad t_{k}>0, \quad \sum t_{k}=2(1-\alpha) \quad(k=1,2, \ldots, n) .
$$

Then there is a complex-valued functional I on $S^{*}(\alpha)$ which is continuous and Fréchet differentiable and such that the equation

$$
\operatorname{Re} I\left(f_{0}\right)=\max \left\{\operatorname{Re} I(f): f \in S^{*}(\alpha)\right\}
$$

holds for a function $f_{0}$ in $S^{*}(\alpha)$ if and only if $f_{0} \in G$.

Proof. Let $\mu$ denote the mapping from $S^{*}(\alpha)$ onto $B$ obtained as the 
composite of the mappings given by equations (35), (32) and (27). By our earlier remarks about the distinguished subsets of $S^{*}(\alpha), P$ and $B$, it follows that each function in the set $F=\mu(G)$ is a finite Blaschke product of degree at most $n-1$. Also, $F$ is closed and so Theorem 2 is applicable. Therefore, there is a functional $J$ on $A$ which is continuous and Fréchet differentiable and so that $\operatorname{Re} J\left(\phi_{0}\right)=\max \{\operatorname{Re} J(\phi): \phi \in B\}$ holds for a function $\phi_{0}$ in $B$ if and only if $\phi_{0} \in F$.

Let the functional $I$ be defined on $S^{*}(\alpha)$ by $I(f)=J(\mu(f))$. Then $I$ is continuous on $S^{*}(\alpha)$ because $J$ is continuous on $A$. Also, $I$ is Fréchet differentiable on $S^{*}(\alpha)$. This can be shown by a computation similar to the one made in Theorem 3 to obtain equation (37.). This results in the formula

$$
\phi^{*}(z)=\phi(z)+\varepsilon\left\{\frac{f(z) g^{\prime}(z)-g(z) f^{\prime}(z)}{\left[z f^{\prime}(z)+(1-2 \alpha) f(z)\right]^{2}}\right\}(2-2 \alpha)+o(\varepsilon) .
$$

Again, the coefficient of $\varepsilon$ is well defined as long as $g \in A$ and $g(0)=0$. Thus, the remaining details concerning the extension of the suitable functional to all of $A$ are the same as those already given for Theorem 3. The conclusion concerning equation (42) is equivalent to the similar fact concerning $J$ and $F$ as $\mu$ is one-to-one.

REMARKS. Results similar to Theorems 3 and 4 are valid for several familiar classes of (regular or meromorphic) functions that are in one-to-one correspondence with $B$ (or, equivalently, with $S^{*}$ ). A main concern is to determine when such correspondences map a Fréchet differentiable functional on one set to a Fréchet differentiable functional on another set. This occurs, for example, when the map between the classes is given by an analytic function of a finite number of continuous linear operators. For the results similar to Theorem 3 the functional needs to avoid a "trivial" form. For the class $P$, this corresponds to the assumption that at the extremal function $p$, the Fréchet derivative does not have the form $I(p, q)=a q(0)$.

4. The support points of $S^{*}(\alpha)$. We shall show that each support point of $S^{*}(\alpha)$ has the form

$$
f(z)=z /(1-x z)^{2(1-\alpha)} \quad(|x|=1) .
$$

We also shall prove a result which is a converse of this fact. It is similar to the previous considerations in that it determines what sets can be the solution sets over $S^{*}(\alpha)$ with respect to continuous linear functionals. Our arguments are quite similar to those given in [3, pp. 101-103] to prove such results when $\alpha=0$. A critical step in this argument for general $\alpha$ will depend on the following lemma.

LEMMA 8. Suppose that $f \in S^{*}(\alpha)$ and $f$ does not have the form given by 
equation (43). Then there are positive numbers $M$ and $\varepsilon$ so that

$$
|f(z)|<M /(1-|z|)^{2(1-\alpha)-\varepsilon} \quad(|z|<1) .
$$

In the case $\alpha=0$, Lemma 8 is a consequence of a result of $\mathrm{Ch}$. Pommerenke [20, Theorem 1]. We shall give an independent proof of this fact based on ideas of subordination. At the same time, we develop similar facts about $K(\alpha)$.

LEMMA 5. Suppose that $f \in K$ and $f$ does not have the form

$$
f(z)=z /(1-x z) \quad(|x|=1) .
$$

Then there are positive constants $M$ and $\varepsilon$ so that

$$
|f(z)|<M /(1-|z|)^{1-e} \quad(|z|<1) .
$$

Proof. It is easy to verify that the only functions in $A$ that map $\Delta$ one-to-one onto a half-plane and have the normalizations $f(0)=0$ and $f^{\prime}(0)=1$ are the functions defined by equation (45). Therefore, if $f \in K$ and $f$ does not have that form, then $f(\Delta)$ is a convex domain which is neither a half-plane nor $\mathbf{C}$. This implies that $f(\Delta)$ has at least two support lines and so $f(\Delta)$ is contained in either an angular wedge with opening less than $\pi$ or in a parallel strip region. In either situation $f$ is subordinate to a suitable function $g$ which maps $\Delta$ one-to-one onto the wedge or the strip.

In the case that $f(\Delta)$ is contained in a wedge, we may let $g(z)=h((z+$ $\left.\zeta) /\left(1+\bar{\zeta}_{z}\right)\right)$ where $h(z)=a[(1+z) /(1-z)]^{\beta}+b$ and $|\zeta|<1,|a|=1$ and $0<\beta<1$. The number $\beta$ produces the suitable opening and the numbers $a$ and $b$ are chosen so that $h(\Delta) \supset f(\Delta)$. Finally, $\zeta$ is determined by the condition $h(\zeta)=0$. The inequality

$$
\left|\frac{z+\zeta}{1+\bar{\zeta}_{z}}\right|<\frac{|z|+|\zeta|}{1+|\zeta||z|} \quad(|z|<1,|\zeta|<1)
$$

implies that there are two positive numbers $M$ and $\varepsilon$ so that

$$
|g(z)|<M /(1-|z|)^{1-\varepsilon} \quad(|z|<1) .
$$

Since $f$ is subordinate to $g$ in $\Delta$ it follows that $\max _{|z|<r}|f(z)|<\max _{|z|<r}|g(z)|$ for every $r(0<r<1)[19$, p. 227]. Therefore, inequality (47) implies result (46).

In the case that $f(\Delta)$ is contained in a strip, we may choose $h$ in the form

$$
h(z)=a \log \left[\frac{1+z}{1-z}\right]+b,
$$

where $|a|$ is determined to produce the appropriate width of the strip $h(\Delta)$. Again, we let $g(z)=h((z+\zeta) /(1+\bar{\zeta} z))$ where $\zeta$ is determined by $h(\zeta)=0$. 
The argument presented in the previous case is applicable and it yields the inequality $|f(z)|<N \log (1-|z|)^{-1}$ for a positive constant $N$. Thus, in this case, inequality (46) holds for every number $\varepsilon, 0<\varepsilon<1$.

LeMma $6[16$, p. 117]. Suppose that $f$ is analytic in $\Delta$ and there are positive constants $M$ and $\gamma$ so that

$$
|f(z)|<M /(1-|z|)^{\gamma} \quad(|z|<1) .
$$

Then there is a positive constant $N$ (depending on $M$ and $\gamma$ ) so that

$$
\left|f^{\prime}(z)\right|<N /(1-|z|)^{\gamma+1} \quad(|z|<1) .
$$

LEMMA 7. If $f \in S^{*}$ and $f$ does not have the form

$$
f(z)=z /(1-x z)^{2} \quad(|x|=1),
$$

then there are positive constants $N$ and $\varepsilon$ so that

$$
|f(z)| \leqslant N /(1-|z|)^{2-\varepsilon} \quad(|z|<1) .
$$

Proof. This is an easy consequence of Lemmas 5 and 6 and the fact that the relation $g(z)=\int_{0}^{z}[f(w) / w] d w$ gives a one-to-one correspondence between $S^{*}$ and $K$.

Proof of Lemma 8. Suppose that $f \in S^{*}(\alpha)$ and $f$ does not have the form of equation (43). The function $g$ defined by $g(z)=z[f(z) / z]^{1 /(1-\alpha)}$ belongs to $S^{*}$ and it does not have the form of equation (50). Thus estimate (51) applies to $g$. By choosing $r_{0}$ so that $0<r_{0}<1$ and considering the two sets $|z|<r_{0}$ and $r_{0}<|z|<1$, we conclude that the same kind of estimate applies to $g(z) / z$. Since $|f(z)|=\left|z[g(z) / z]^{1-\alpha}\right|<|g(z) / z|^{1-\alpha}$ this provides a bound on $f$ given by the form of inequality (44).

The usual one-to-one correspondence between $K(\alpha)$ and $S^{*}(\alpha)$ and an integration of the bound provided by Lemma 8 yields the following result. For each fixed value of $\alpha$ greater than $\frac{1}{2}$ the family $K(\alpha)$ is uniformly bounded [22].

LEMMA 9. Suppose that $f \in K(\alpha)$. If $\alpha<\frac{1}{2}$ and $f$ does not have the form

$$
f(z)=\frac{1}{(1-2 \alpha) x}\left\{\frac{1}{(1-x z)^{1-2 \alpha}}-1\right\} \quad(|x|=1),
$$

then there are positive constants $Q$ and $\varepsilon$ so that

$$
|f(z)| \leqslant Q /(1-|z|)^{1-2 \alpha-e} \quad(|z|<1) .
$$

If $\alpha=\frac{1}{2}$ and $f$ does not have the form

$$
f(z)=-x^{-1} \log (1-x z) \quad(|x|=1),
$$

then there is a positive constant $Q$ so that 


$$
|f(z)|<Q \quad(|z|<1) .
$$

THEOREM 5. Each support point of $S^{*}(\alpha)$ has the form

$$
f(z)=z /(1-x z)^{2(1-\alpha)} \quad(|x|=1) .
$$

Proof. Suppose that $J$ is a continuous, linear functional on $A$. Then there is a sequence $\left\{b_{n}\right\}$ of complex numbers so that equation (19) holds and $J$ operates accordingly to equation (20). We also assume that $J$ is not constant on $S^{*}(\alpha)$. This is equivalent to the assertion that $b_{n} \neq 0$ for some $n>2$. This can be seen by considering the functions $f(z)=z+a_{n} z^{n}\left(a_{n} \neq 0\right)$ which belong to $S^{*}(\alpha)$ for $\left|a_{n}\right|$ sufficiently small.

The set of extreme points of the closed convex hull of $S^{*}(\alpha)$, denoted $\mathcal{E} \operatorname{ISS}^{*}(\alpha)$, consists of the functions $f$ described by equation $(56)[4, \mathrm{p} .417]$. The $n$th coefficient of the power series for this function $f$ is given by $a_{n}=c_{n} x^{n-1}$, where

$$
c_{n}=\frac{(2-2 \alpha)(3-2 \alpha) \ldots(n-2 \alpha)}{(n-1) !} \quad(n=2,3, \ldots) .
$$

Therefore, equation (20) implies that

$$
J(f)=b_{1}+\sum_{n=2}^{\infty} b_{n} c_{n} x^{n-1}
$$

It is easy to show that $c_{n}^{1 / n} \rightarrow 1$, and thus equation (19) implies that

$$
\lim \sup \left|b_{n} c_{n}\right|^{1 / n}=\lim \sup \left|b_{n}\right|^{1 / n}<1 .
$$

Consequently, equation (57) defines a function, denoted by $h$, that is analytic for $|x|<1$. Moreover, $h$ is not constant.

The argument given in [3, p. 100] shows that if $F$ is the set of all functions $f_{0}$ in $\operatorname{TS}^{*}(\alpha)$ such that

$$
\operatorname{Re} J\left(f_{0}\right)=\max \left\{\operatorname{Re} J(f): f \in \mathcal{H} S^{*}(\alpha)\right\},
$$

then there is a positive integer $m$ so that $F$ is the same as the set of functions $f_{0}$ given by

$$
f_{0}=\sum_{k=1}^{m} t_{k} f_{k}
$$

where

$$
f_{k}(z)=\frac{z}{\left(1-x_{k} z\right)^{2(1-\alpha)}}
$$

and

$$
0<t_{k} \leqslant 1, \quad\left|x_{k}\right|=1, \quad \sum_{k=1}^{n} t_{k}=1 .
$$


In particular, this shows that the only functions $f_{0}$ in $S^{*}(\alpha)$ that maximize $\operatorname{Re} J(f)$ over $S^{*}(\alpha)$ have the form prescribed by equations (59)-(61). If $t_{k} \neq 0$ for some $k$, then by letting $z$ tend to $\bar{x}_{k}$ radially we see that the function $f_{0}$ of equation (59) does not satisfy inequality (44) of Lemma 8 (with $M>0$ and $\varepsilon>0)$. Therefore, the only functions given by equations (59) $-(61)$ which also belong to $S^{*}(\alpha)$ are the functions $f_{k}(k=1,2, \ldots, m)$. These are the only functions in $S^{*}(\alpha)$ that satisfy $\operatorname{Re} J\left(f_{0}\right)=\max \left\{\operatorname{Re} J(f): f \in S^{*}(\alpha)\right\}$.

THEOREM 6. Each support point of $K(\alpha)$ has the form

$$
f_{0}(z)=\left\{\begin{array}{l}
\frac{1}{(1-2 \alpha) x}\left[\frac{1}{(1-x z)^{1-2 \alpha}}-1\right], \text { if } \alpha \neq \frac{1}{2}, \\
-x^{-1} \log (1-x z), \text { if } \alpha=\frac{1}{2},
\end{array}\right.
$$

where $|x|=1$.

Proof. Let the functional $J$ be continuous and linear on $A$ and not constant on $K(\alpha)$, and let $J$ be given by the sequence $\left\{b_{n}\right\}$ described by equations (19) and (20). Define the sequence $\left\{b_{n}^{\prime}\right\}$ by $b_{0}^{\prime}=b_{0}$ and $b_{n}^{\prime}=b_{n} / n$ for $n=1,2, \ldots$ and thus $\lim \sup \left|b_{n}^{\prime}\right|^{1 / n}=\lim \sup \left|b_{n}\right|^{1 / n}<1$. Therefore, the functional $J^{\prime}$, defined by $J^{\prime}(f)=\sum_{n=0}^{\infty} b_{n}^{\prime} a_{n}$ when $f \in A$ and $f(z)=$ $\sum_{n=0}^{\infty} a_{n} z^{n}$, is continuous and linear on $A$ [27]. Also, $J^{\prime}$ is nonconstant on $S^{*}(\alpha)$.

The mapping from $K(\alpha)$ to $S^{*}(\alpha)$ defined by $g(z)=z f^{\prime}(z)$ is a linear homeomorphism, and for this mapping $J^{\prime}(g)=J(f)$. Theorem 5 is applicable to the functional $J^{\prime}$ and the conclusions are equivalent to the resuults of this theorem.

Using the facts we have established about $S^{*}(\alpha)$ it is quite easy to prove the following converses of Theorems 5 and 6 . The arguments are similar to those presented in [3, pp. 101-103] for the case $\alpha=0$.

THEOREM 7. Let $m$ functions $f_{k}$ be given by $f_{k}(z)=z /\left(1-x_{k} z\right)^{2(1-\alpha)}$ where $\alpha<1$ and the complex numbers $x_{k}$ satisfy $\left|x_{k}\right|=1(k=1,2, \ldots, m)$. There is a continuous, linear functional $J$ on $A$ so that the set of functions $f_{0}$ in $S^{*}(\alpha)$ that satisfy $\operatorname{Re} J\left(f_{0}\right)=\max \left\{\operatorname{Re} J(f): f \in S^{*}(\alpha)\right\}$ is the set $\left\{f_{1}, f_{2}, \ldots, f_{m}\right\}$.

THEOREM 8. Let $m$ functions $\left\{f_{k}\right\}$ be given by

$$
f_{k}(z)=\left\{\begin{array}{l}
\frac{1}{(1-2 \alpha) x_{k}}\left[\frac{1}{\left(1-x_{k} z\right)^{1-2 \alpha}}-1\right], \quad \text { if } \alpha \neq \frac{1}{2}, \\
-\left(x_{k}\right)^{-1} \log \left(1-x_{k} z\right), \quad \text { if } \alpha=\frac{1}{2},
\end{array}\right.
$$

where $\alpha<1$ and the complex numbers $x_{k}$ satisfy $\left|x_{k}\right|=1(k=1,2, \ldots, m)$. 
There is a continuous, linear functional $J$ on $A$ such that the set of functions $f_{0}$ in $K(\alpha)$ that satisfy $\operatorname{Re} J\left(f_{0}\right)=\max \{\operatorname{Re} J(f): f \in K(\alpha)\}$ is the set $\left\{f_{1}, f_{2}, \ldots, f_{m}\right\}$.

\section{REFERENCES}

1. N. I. Akhiezer, The classical moment problem and some related questions in analysis, Fizmatgiz, Moscow, 1961; English transl., Hafner, New York, 1965. MR 27 \#4028; 32 \# 1518.

2. D. A. Brannan, J. G. Clunie and W. E. Kirwan, On the coefficient problem for functions of bounded boundary rotation, Ann. Acad. Sci. Fenn. A I No. 523 (1973), 18 pp. MR 49 \#3108.

3. L. Brickman, T. H. MacGregor and D. R. Wilken, Convex hulls of some classical families of univalent functions, Trans. Amer. Math. Soc. 156 (1971), 91-107. MR 43 \#494.

4. L. Brickman, D. J. Hallenbeck, T. H. MacGregor and D. R. Wilken, Convex hulls and extreme points of families of starlike and convex mappings, Trans. Amer. Math. Soc. 185 (1973), 413-428 (1974). MR 49 \#3102.

5. C. Caratheodory, Über den Variabilitätsbereich der Fourier'schen Konstanten von positiven harmonischen Funktionen, Rend. Circ. Mat. Palermo 32 (1911), 193-217.

6. __ Funktionentheorie, Bd. 2, Birkhäuser, Basel, 1950; English transl., Theory of functions of a complex variable, Vol. 2, Chelsea, New York, 1954. MR 12, 248; 16346.

7. P. Dienes, The Taylor series, Dover, New York, 1957. MR 19, 735.

8. N. Dunford and J. T. Schwartz, Linear operators. I: General theory, Interscience, New York and London 1958. MR 22 \#8302.

9. P. L. Duren, Theory of $H^{p}$ spaces, Academic Press, New York, 1970. MR 42 \#3552.

10. M. Golubitsky and V. Guillemin, Stable mappings and their singularities, Springer-Verlag, New York, 1973. MR 49 \#6269.

11. G. M. Goluzin, Geometric theory of functions of a complex variable, 2nd ed., "Nauka", Moscow, 1966; English transl., Transl. Math. Monographs, vol. 26, Amer. Math. Soc., Providence, R. I., 1969. MR 36 \#2793; 40 \#308.

12. E. Hille and R. S. Phillips, Functional analysis and semi-groups, Amer. Math. Soc. Collog. Publ., vol. 31, rev. ed., Amer. Math. Soc., Providence, R. I., 1957. MR 19, 664.

13. J. A. Hummel, Extremal problems in the class of starlike functions, Proc. Amer. Math. Soc. 11 (1960), 741-749. MR 22 \#11133.

14. W. E. Kirwan and G. Schober, On extreme points and support points for some families of univalent functions, Duke Math. J. (to appear).

15. T. H. MacGregor, Applications of extreme-point theory to univalent functions, Michigan Math. J. 19 (1972), 361-376. MR 47 \#447.

16. Rotations of the range of an analytic function, Math. Ann. 201 (1973), 113-126. MR 48 \#6390.

17. __ Hull subordination and extremal problems for starlike and spirallike mappings, Trans. Amer. Math. Soc. 183 (1973), 499-510. MR 49 \#3104.

18. A. J. Macintyre and W. W. Rogosinski, Extremum problems in the theory of analytic functions, Acta Math. 82 (1950), 275-325. MR 12, 89.

19. Z. Nehari, Conformal mapping, McGraw-Hill, New York, 1952. MR 13, 640.

20. Ch. Pommerenke, On starlike and convex functions, J. London Math. Soc. 37 (1962), 209-224. MR 25 \# 1279.

21. P. Porcelli, Linear spaces of analytic functions, Rand McNally, Chicago, 1966. MR 41 \#4219.

22. M. S. Robertson, On the theory of univalent functions, Ann. of Math. (2) 37 (1936), 374-408.

23. W. W. Rogosinski, Über positive harmonische Entwicklungen und typisch-reelle Potenzreihen, Math. Z. 35 (1932), 93-121.

24. J. Schur, Über Potenzreihen, die im Innern des Einheitskreises beschränkt sind, J. Math. 147 (1917), 205-232. 
25. __ Über Potenzreihen, die im Innern des Einheitskreises beschränkt sind, J. Math. 148 (1918), 122-145.

26. A. E. Taylor, Introduction to functional analysis, Wiley, New York; Chapman and Hall, London, 1958. MR 20 \#5411.

27. O. Toeplitz, Die linearen vollkommenen Räume der Funktionentheorie, Comment. Math. Helv. 23 (1949), 222-242. MR 11, 372.

Departiant of Mathematics, State University of New York, Albany, New York 12222 (Current address of T. H. MacGregor)

Current address (P. C. Cochrane): Department of Mathematics, Bloomsburg State College, Bloomsburg, Pennsylvania 17815 